\title{
Crystal-Liquid Phase Relations in Silicon at Negative Pressure
}

\author{
Mark Wilson and Paul F. McMillan \\ Department of Chemistry, University College London, 20 Gordon Street, London WC1H OAJ, United Kingdom
}

(Received 21 December 2002; published 4 April 2003)

\begin{abstract}
The stable and metastable melting relations for silicon in the diamond and $\mathrm{Si}_{136}$ clathrate-II structures at positive and negative pressures are calculated by molecular dynamics computer simulation. The simulated liquid and crystalline clathrates undergo cavitation at approximately -3 and $-12 \mathrm{GPa}$. Between these limits a stretched crystal would transform directly to gas in response to a mechanical instability. Most importantly, the clathrate-II crystal becomes thermodynamically stable over the diamond at negative pressure below $-1 \mathrm{GPa}$ at the melting point. $\mathrm{Si}_{136}$ should then crystallize from a slightly stretched liquid, which would have the same volume as a diamond-structure crystal.
\end{abstract}

DOI: $10.1103 /$ PhysRevLett.90.135703

PACS numbers: 64.70.Dv, 68.08.De

A new class of Si-based materials for future development is derived from the "semiconductor clathrates." These are open-framework structures based on $\mathrm{Si}, \mathrm{Ge}$, $\mathrm{Ga}$, etc., in slightly distorted tetrahedral coordination [1] and are isotypic with clathrate hydrates. In the semiconductor clathrates, metal atoms (typically $\mathrm{Na}, \mathrm{Ba}, \mathrm{K}$, or $\mathrm{Sr}$ ) are encapsulated, and their electronic behavior depends upon the guest atom and framework chemistry. The clathrates have low thermal conductivity, and they have been proposed as a new class of thermoelectric materials $[2,3]$. Two frameworks (clathrate-I and -II) have been studied in detail [4-7]. Type I clathrates $\left(\mathrm{Na}_{8} \mathrm{Si}_{46}\right.$ or $\left.\mathrm{Sr}_{8} \mathrm{Ga}_{16} \mathrm{Ge}_{30}\right)$ contain dodecahedral (20-atom) and tetrakaidecahedral (24-atom) cages. Type II clathrates $\left(\mathrm{Cs}_{8} \mathrm{Na}_{16} \mathrm{Si}_{136}\right.$ or $\mathrm{Na}_{x} \mathrm{Si}_{136}$, where $0<x<24$ ) contain guest atoms distributed among dodecahedral and hexakaidecahedral (28-atom) cages.

The framework structure contains planar five- and six-membered rings of $\mathrm{Si}$ atoms: the forced planarity of the six-rings results in a slight distortion of the tetrahedral units, and the band gap is opened up. Ab initio calculations indicate that a guest-free clathrate $\left(\mathrm{Si}_{136}\right)$ would have an indirect band gap approximately twice that of diamond-structured silicon $(1.11 \mathrm{eV}$ at $300 \mathrm{~K}$ [8]) [9]. Complete removal of $\mathrm{Na}$ atoms from the type II clathrate $\mathrm{Na}_{x} \mathrm{Si}_{136}$ was recently achieved [10], giving a new open-framework form of elemental silicon. The band gap was determined by conductivity and optical measurements to be approximately $1.9 \mathrm{eV}$. It would be useful to develop this material for optoelectronic applications. The new material also has a remarkably low lattice thermal conductivity, comparable with amorphous$\mathrm{SiO}_{2}$, that could lead to additional electronics applications $[2,11,12]$.

It is important to develop synthesis routes to this new form of elemental Si that will permit high-purity crystalline material to be grown. Potential routes involving vapor-phase synthesis have been suggested from laser ablation/cluster deposition, or from observations on $\mathrm{Si}$ crystal growth in He atmospheres following sputtering.
However, these approaches lead to amorphous aggregates or nanorods of diamond-structured silicon, with only a hint of crystalline clathrate [13]. Recently, based upon results of molecular dynamics (MD) simulations, Motooka et al. [14] and Miranda and Antonelli [15] proposed a new approach to the liquid-state epitaxy of Si clathrate. They calculated stable and metastable relations between liquid $\mathrm{Si}$, diamond-structured phase, and clathrate structures I and II (i.e., $\mathrm{Si}_{46}$ and $\mathrm{Si}_{136}$ ) and observed that clathrate melting occurred below that of the stable structure. This result is in agreement with experimental determination for $\operatorname{Si}_{136}\left(T_{m} \sim 1200^{\circ} \mathrm{C}\right)$ [16]. It was proposed that crystalline clathrates could be obtained by epitaxial growth on a clathrate-structured seed from the supercooled liquid, or by controlled crystallization from solid amorphous Si. Supercooled liquid $\mathrm{Si}$ and Ge are known from levitation experiments [17], however, to undergo rapid homogeneous crystallization of the diamond-structured phase in response to spinodal fluctuations associated with an underlying liquidliquid phase transition [18,19]. Solid $a$-Si samples usually contain substantial hydrogen, which promotes recrystallization of nanocrystalline silicon [20]. These suggested growth strategies for clathrate Si are likely to encounter serious experimental obstacles. However, the results indicate that an understanding of the metastable phase diagram, in particular, at negative pressure, is of considerable interest if manufacturing strategies are to be realized.

In the present work, we pursued investigations of the MD melting of dia-Si and $\mathrm{Si}_{136}$ into the "negative pressure" regime using a simple but reliable model, based upon recent experiments and ab initio theoretical predictions that indicated a stability crossover between the two phases at $-3 \mathrm{GPa}$, at low temperature [21]. Such a tensile regime (extending to at least $-1 \mathrm{GPa}$ ) is readily accessible in semiconductor structures due to latticemismatched epitaxy or during formation/annealing of an $\mathrm{SiO}_{2}$ overlayer [22]. A well-known feature of the $\mathrm{Si}$ phase diagram is its negative $d T_{m} / d P$ melting relation for 
the diamond-structured semiconductor, which implies a maximum at negative pressure $[18,23]$ where the densities of liquid and crystal become equal. The MD simulations indicate a crossover occurs in the melting curves of dia-Si and $\mathrm{Si}_{136}$ at slightly negative pressures, resulting in the possibility of stable clathrate crystal growth when the system is subjected to tensile stresses. Although condensed phases under negative pressure are always metastable to gas, metastable Si liquids can be maintained to at least $-3 \mathrm{GPa}$ before cavitation [24], and the crystalline clathrate is preserved to at least $-11 \mathrm{GPa}$ (this work).

The silicon is modeled using a Stillinger-Weber threebody (SW3) potential [25] known to reliably reproduce a range of known liquid and crystal properties [26]. The SW3 potential is chosen over possible alternatives such as that developed by Tersoff (used to study the phase stabilities at zero pressure $[14,15])$ as it is known to give better agreement with the experimental melting relations of the diamond structure [27]. Both clathrate-II and diamond structures are constructed exclusively from tetrahedral units so that properties of these crystals may be reproduced with reasonable accuracy.

Figure 1 shows the $0 \mathrm{~K}$ energy/volume curves for the diamond and $\mathrm{Si}_{136}$ crystals compared with the results of density-functional calculations in both the local density approximation (LDA) and the gradient corrected approximation (GGA) [9]. The experimental volumes are also indicated in the figure as arrows. The equilibrium volumes of both simulated crystal phases are in excellent agreement with both experiment and electronic structure calculation. The energy difference between the two crystals at their respective minima is $4.2 \mathrm{~kJ} \mathrm{~mol}^{-1}$ for the SW3 potential compared with values of $7.4 \mathrm{~kJ} \mathrm{~mol}^{-1}$ (LDA) and $5.3 \mathrm{~kJ} \mathrm{~mol}^{-1}$ (GGA). The minimum energy lattice parameters for the SW3, LDA, and GGA calcula-

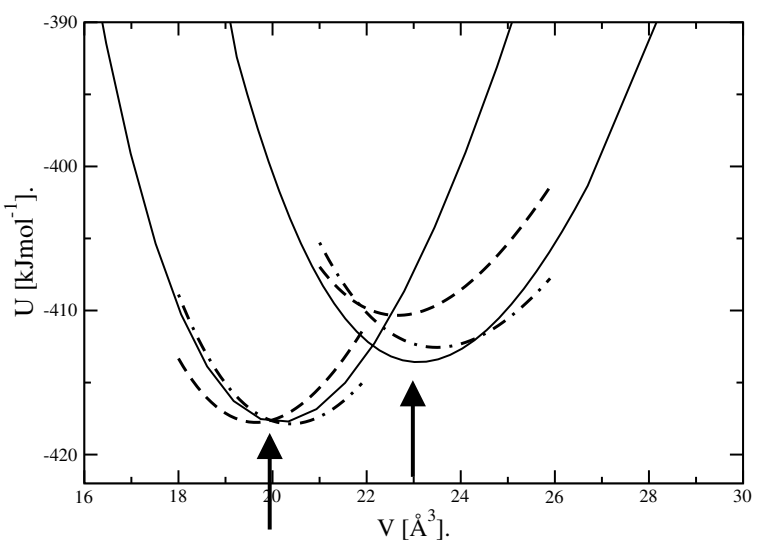

FIG. 1. Energy/volume curves for the diamond and $\mathrm{Si}_{136}$ structures calculated using the SW3 potential (present work, solid lines) and the LDA (dashed lines) and GGA (dot-dashed lines) density functionals [9]. The LDA and GGA curves have been shifted to agree with the energy minimum value for the SW3 diamond calculation. The arrows indicate the experimental volumes for the two structures. tions are $5.43,5.40$, and $5.46 \AA$, respectively (experimental value $5.43 \AA[2])$ for the diamond structure, and 14.65 , 14.55 , and $14.73 \AA$ (experimental value $14.62 \AA$ [10]) for the $\mathrm{Si}_{136}$. The $\mathrm{SW} 3$ potential calculations predict a $0 \mathrm{~K}$ diamond $\rightarrow \mathrm{Si}_{136}$ pressure-driven phase transition at $\sim-2.4 \mathrm{GPa}$ compared with $\sim-4.0 \mathrm{GPa}(\mathrm{LDA})$ and $\sim-2.7 \mathrm{GPa}(\mathrm{GGA})$.

The interfaces are constructed by combining two separate simulation cells [28]. Simulations are performed on both the crystals and liquid at both constant temperature and pressure (NPT ensemble) in cubic simulation cells at state points corresponding to the estimated coexistence temperature in order to generate equilibrium configurations. The constant temperature and pressure constraints are applied using Nosé-Hoover thermostats and barostats as developed by Martyna and co-workers [29]. These cells are then combined to give a tetragonal simulation cell elongated along a single axis with the liquid/solid interfaces set up so as to be perpendicular to the crystal [100] direction. These cells are periodically repeated in three dimensions giving an infinite series of liquid/solid slabs containing two interfaces per simulation cell. The diamond/liquid and $\mathrm{Si}_{136}$ /liquid interfaces contain 2000 and 2428 atoms, respectively. Simulations are then performed in the NPT ensemble using the atom velocities carried over from the separate crystal and liquid simulations. Once reequilibrated the phase diagram is mapped by performing simulations on both the diamond/liquid and $\mathrm{Si}_{136} /$ liquid systems over a range of temperatures and pressures. At each pressure and temperature crystal growth or melting is monitored. Both systems are found to show a significant crystallization front extending into the liquid phase when undercooled. The temperature is then systematically varied until the liquid and crystal are in equilibrium [30] as monitored by reference to the time evolution of the system energy and the density profiles (see below).

Figure 2(a) shows molecular graphics "snapshots" of two equilibrium liquid/crystal interfaces for the diamond/liquid and $\mathrm{Si}_{136}$ /liquid interfaces at a pressure of $\sim-1.5 \mathrm{GPa}$ and a temperature of $1750 \mathrm{~K}$ after $\sim 0.25 \mathrm{~ns}$ of dynamics. In both cases the highly ordered crystalline regions are clearly visible with the approximate locations of the two interfaces indicated by arrows. A small number of atoms originally in the bulk crystal configuration have diffused into the liquid region. This highlights the fact that the atoms in the liquid are still diffusing on the simulation time scale. Figure 2(b) shows equilibrium density profiles for the same two systems averaged over a time of $100 \mathrm{ps}$. The density profiles are generated by calculating the atom distributions in slabs of thickness $0.13 \AA$ perpendicular to the [100] direction. The profiles shown are divided by the atom coordination number (defined by calculating the number of nearest neighbors about a given atom within a cutoff of $2.90 \AA$ A). The curves shown include the total density profile and individual density profiles for four-, five-, and 

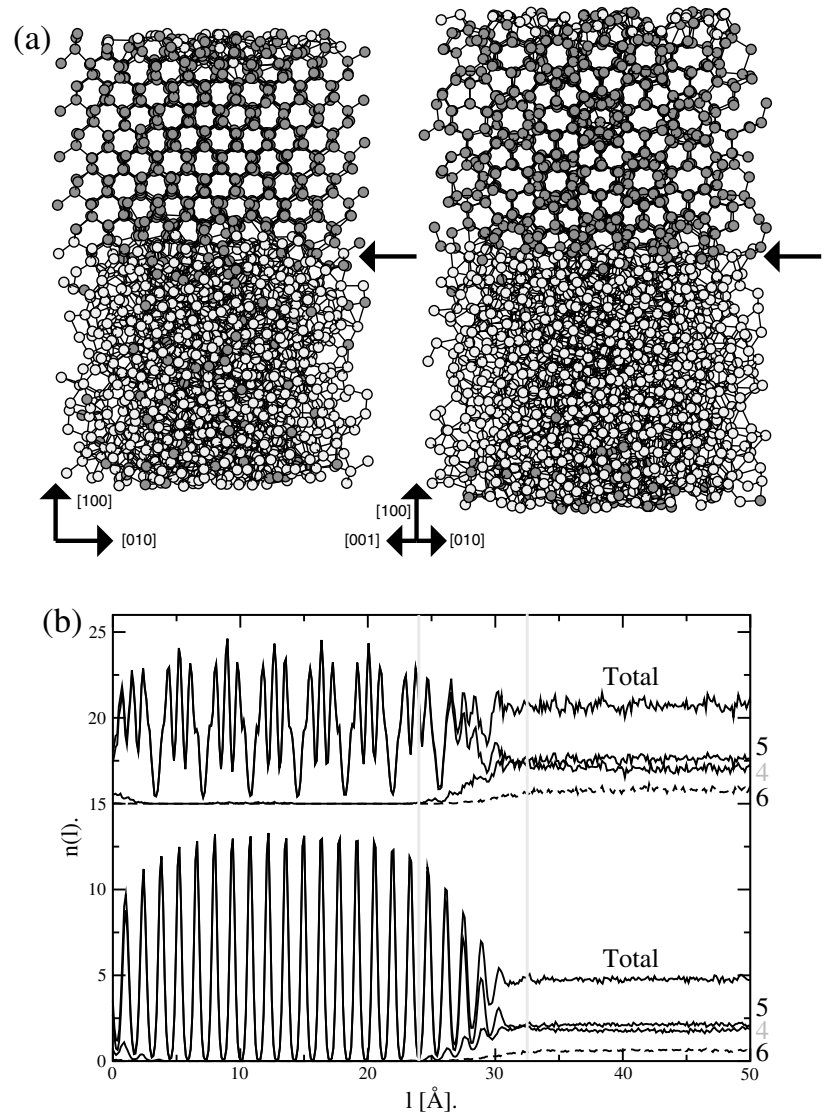

FIG. 2. (a) Molecular graphics "snapshots" of the diamond/liquid (left) and $\mathrm{Si}_{136}$ /liquid interfaces (right) at $p=$ $-1.5 \mathrm{GPa}$ and $T=1750 \mathrm{~K}$ oriented with respect to the crystals as indicated. The light circles represent the atoms originally in the bulk liquid configuration and the dark circles those atoms originally in the crystal. The approximate locations of the central interfaces are indicated by the arrows. (b) Density profiles for the two systems [ $\mathrm{Si}_{136} /$ liquid (upper curves) shifted by 15 units along the $y$ axis] shown in (a) calculated over $\sim 100$ ps of dynamics. The approximate interface region is highlighted by the vertical dashed lines. Key (indicated on the figure): black, total atom profiles; grey, four coordinate; black, five coordinate; dashed line, six coordinate. The lower profile for the five-coordinate sites shows the characteristic defect oscillations discussed in the text.

six-coordinated atoms. Strong periodic oscillations are evident for $l<30 \AA$ indicative of the retained crystalline region that is clear in Fig. 2(a). The difference in the oscillation periodicities simply reflects the structure of the diamond and clathrate-II crystals in the [100] direction. A bulklike liquid region is clearly observed at $l>$ $35 \AA$ in which the distribution of the atom coordination environments become equivalent (as is expected). Across the liquid/solid interface a significant change in atom coordination number occurs. Both solid phases are constructed from only four-coordinate atoms. In the liquid state, however, four-, five-, and six-coordinate atoms are present [26]. The density profiles also allow us to identify interfacial regions in which the atoms are in coordination environments intermediate between those typical of the liquid and crystal. In both cases these regions (highlighted in the figure) are of the order of $\sim 10 \AA$ in length. The interfacial regions show a significantly different structure for the two crystals. For the $\mathrm{Si}_{136} /$ liquid interface the number of both five- and six-coordinate sites increases monotonically from zero (in the crystal slab) to their bulk values in the liquid slab. For the diamond/liquid interface, however, the density profile for the five-coordinate sites shows an unexpected characteristic oscillation. This corresponds to the emergence of $\mathrm{Si}$ atoms in specific defect sites available in the diamond lattice but not $\mathrm{Si}_{136}$. The diamond lattice consists of two interpenetrating fcc sublattices, with one occupying half of the available tetrahedral holes in the other; the fivecoordinate defect site corresponds to an atom sitting in one of the alternative tetrahedral sites at the crystalliquid interface. In fact, the atom position is slightly distorted from the ideal lattice site as evidenced by the fact that the peaks in the five-coordinate density profile are slightly offset (by $\sim 0.15 \AA$ ) when compared with the four-coordinate profile.

Figure 3 shows the liquid/crystal phase diagram for the diamond and $\mathrm{Si}_{136}$ crystal phases. At positive pressure the melting line of the clathrate lies at significantly lower temperature than that of the diamond form, in agreement

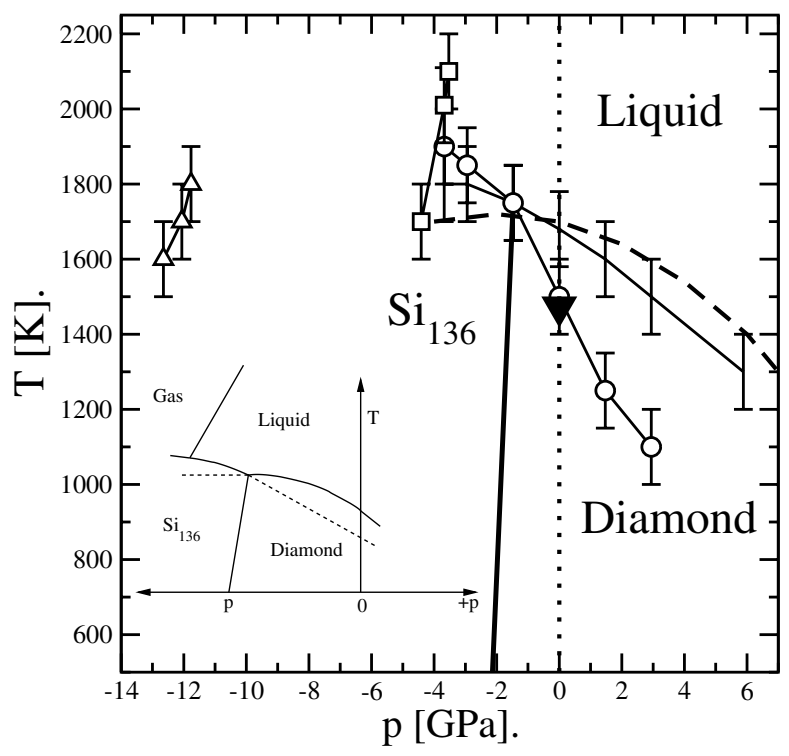

FIG. 3. Phase diagram for the liquid/diamond/ $/ \mathrm{Si}_{136}$ system obtained from the present SW3 empirical potential calculations compared with experiment [16]. Key to the phase boundaries: no symbol, diamond/liquid; circles, $\mathrm{Si}_{136}$ /liquid; light (grey) line, $\mathrm{Si}_{136} /$ diamond, cavitation limits; squares, liquid; triangles, $\mathrm{Si}_{136}$. The dashed line represents the experimental diamond/liquid melting curve and the downward solid triangle the experimental melting point of the $\mathrm{Si}_{136}$ phase at zero pressure. The inset shows a schematic of the final phase diagram. 
with experiment. At zero pressure the melting point of the $\mathrm{Si}_{136}$ clathrate lies $180 \mathrm{~K}$ below that of the diamond, compared with an experimental value of $214 \mathrm{~K}$ [16], and values of 150 and $100 \mathrm{~K}$ obtained using the Tersoff $[14,15,31]$ and the environment-dependent interatomic $[15,32]$ potential models, respectively. At negative pressure (in the tensile regime) the clathrate structure becomes thermodynamically stabilized relative to the diamond, and the clathrate melting curve lies at higher temperature than that of diamond. The crossover point (at which state point the diamond, clathrate, and liquid would coexist) is calculated to be at $p=-1.5 \mathrm{GPa}$ and $T=1750 \mathrm{~K}$ compared with that estimated from experiment of $p=-2.5 \mathrm{GPa}$ and $T=1710 \mathrm{~K}$. We should point out that at negative pressure liquid and solid states are metastable with respect to the vapour. Figure 3 shows the metastability limits for the stretched crystal compared with the liquid. The liquid cavitates at $\sim-3 \mathrm{GPa}$ while the crystal remains stable under tension to around $-11 \mathrm{GPa}$.

The present MD simulations extend our knowledge of the metastable relations between liquid silicon, the diamond-structured semiconductor form, and the "expanded-framework" clathrate form $\mathrm{Si}_{136}$ into the negative pressure regime. "Stretched" liquids are maintained to $-3 \mathrm{GPa}$, within a tensile regime readily accessed by semiconductor growth and processing techniques, and the $\mathrm{Si}_{136}$ crystal is metastable to -8 to $-9 \mathrm{GPa}$. It is interesting to note the difference in relative metastability limits of amorphous and crystalline phases: the crystal contains a single type of $\mathrm{Si}$ coordination environment (slightly distorted tetrahedral) and reaches its spinodal limit via a coherent lattice instability, whereas the liquid has a range of four-, five-, and sixcoordinated sites, and instabilities toward low-density states are generated by sampling multiple minima among potential configurations. As we melt or crystallize $\mathrm{Si}$ under slightly strained conditions, the $\mathrm{Si}_{136} /$ liquid and dia-Si/liquid $d T_{m} / d P$ relations are nearly flat; i.e., the molar volumes are nearly the same. As is evident from the schematic phase diagram shown in the inset to Fig. 3, if the silicon sample can be "slightly stretched" before and during melting, it might be expected from these MD simulations that equilibrium growth of $\mathrm{Si}_{136}$ (or perhaps $\mathrm{Si}_{46}$ ) Si-clathrate crystals will occur from the liquid, as the clathrate phase becomes more thermally stable than and comparable in volume with the $\mathrm{Si}$-diamond structure.

M.W. thanks the Royal Society for support.

[1] C. Cros et al., J. Solid State Chem. 2, 570-581 (1970); G. K. Ramachandran et al., J. Solid State Chem. 145, 716 (1999); S. Bobev and S. C. Sevov, J. Solid State Chem. 153, 92 (2000); A. A. Demkov et al., Phys. Rev. B 53, 11288 (1996).

[2] G. S. Nolas et al., Appl. Phys. Lett. 73, 178 (1998).
[3] J. L. Cohn et al., Phys. Rev. Lett. 82, 779 (1999); B. C. Sales et al., Phys. Rev. B 63, 245113 (2001).

[4] E. Reny et al., J. Mater. Chem. 8, 2839 (1998); A. San-Miguel et al., Phys. Rev. B 65, 054109 (2002).

[5] P. Mélinon et al., Phys. Rev. B 58, 12590 (1998).

[6] G. K. Ramachandran et al., Phys. Rev. B 60, 12294 (1999).

[7] S. Bobev and S. C. Sevov, J. Am. Chem. Soc. 121, 3795 (1999).

[8] J. I. Pankove, Optical Processes in Semiconductors (Prentice-Hall Inc., Englewood Cliffs, NJ, 1971).

[9] G. B. Adams et al., Phys. Rev. B 49, 8048 (1994); J. Dong et al., Phys. Rev. B 60, 950 (1999).

[10] J. Gryko et al., Phys. Rev. B 62, R7707 (2000).

[11] See, for example, S. Saito and A. Oshiyama, Phys. Rev. B 51, 2628 (1995).

[12] G. S. Nolas et al. (to be published).

[13] R. Kamalakaran et al., J. Phys. Condens. Matter 7, L529 (1995); M. Broyer et al., Mater. Sci. Forum 232, 27 (1996); P. Melinon et al., Mater. Sci. Eng. A 217/218, 69 (1996).

[14] K. Moriguchi et al., Phys. Rev. B 64, 195409 (2001); S. Munetoh et al., Phys. Rev. Lett. 86, 4879 (2001); T. Motooka et al., Phys. Rev. B 61, 8537 (2000); S. Munetoh et al., Phys. Rev. B 64, 193314 (2001).

[15] C. A. Miranda and A. Antonelli (to be published).

[16] P. F. McMillan, Nature Materials 1, 19 (2002).

[17] S. A. Ansell et al., J. Phys. Condens. Matter 10, L73-L78 (1998).

[18] S. K. Deb et al., Nature (London) 414, 528-530 (2001).

[19] A. Filipponi and A. Di Cicco, Phys. Rev. B 51, 12322 (1995).

[20] K. Saitoh et al., Appl. Phys. Lett., 71, 3403 (1997).

[21] G. K. Ramachandran et al., J. Phys. Condens. Matter 12, 4013 (2000).

[22] Th. Englert et al., Solid-State Electron. 23, 31 (1980); E. Anastassakis et al., Solid State Commun. 8, 133 (1970); J.T. Fitch et al., J. Vac. Sci. Technol. B 7, 775 (1989).

[23] E. G. Ponyatovsky and O. I. Barkalov, Mater. Sci. Rep. 8, 147-191 (1992).

[24] C. A. Angell et al., J. Non-Cryst. Solids 205-207, 463471 (1999).

[25] F. H. Stillinger and T. A. Weber, Phys. Rev. B 31, 5262 (1985).

[26] W. D. Lüdtke and U. Landman, Phys. Rev. B 37, 4656 (1988); 40, 1164 (1989).

[27] F. F. Abraham and J. Q. Broughton, Phys. Rev. Lett. 56, 734 (1986); U. Landman et al., Phys. Rev. Lett. 56, 155 (1986); U. Landman et al., Phys. Rev. B 37, 4637 (1988); W. D. Lüdtke et al., Phys. Rev. B 37, 4647 (1988).

[28] B. B. Laird and A. D. J. Haymet, Chem. Rev. 92, 1819 (1992).

[29] S. Nosé, J. Chem. Phys. 81, 511 (1984); W. G. Hoover, Phys. Rev. A 31, 1695 (1985); G. J. Martyna et al., J. Chem. Phys. 101, 4177 (1994).

[30] B. J. Jesson and P. A. Madden, J. Chem. Phys. 113, 5935 (2000).

[31] J. Tersoff, Phys. Rev. B 39, 5566 (1989).

[32] J. F. Justo et al., Phys. Rev. B 58, 2539 (1998). 